\title{
Morphological Features and Immunohistochemical Expression of p57Kip2 in Early Molar Pregnancies and Their Relations to the Progression to Persistent Trophoblastic Disease
}

\author{
Marwa Khashaba \\ Mohammad Arafa ${ }^{1}$ \\ Eman Elsalkh ${ }^{1} \cdot$ Reda Hemida ${ }^{2}$ \\ Wagiha Kandil ${ }^{1}$ \\ Department of Pathology, Faculty of Medicine, \\ Port Said University, Port Said; Departments of \\ ${ }^{1}$ Pathology and ${ }^{2}$ Obstetrics and Gynecology, \\ Faculty of Medicine, Mansoura University, \\ Mansoura, Egypt
}

Received: January 31, 2017

Revised: April 19, 2017

Accepted: April 28, 2017

Corresponding Author

Mohammad Arafa, MD, PhD

Department of Pathology, Faculty of Medicine,

Mansoura University, 35516 Mansoura, Egypt

Tel: $+20-50-226-5922$

Fax: +20-50-226-3717

E-mail: marafa8@yahoo.com

\begin{abstract}
Background: Although the morphological features characteristic of products of conception specimens including molar pregnancies are well described, substantial histopathological similarities are observed between the different entities, especially in cases of early pregnancies. Furthermore, there are no current solid criteria that could predict cases with progression to persistent gestational trophoblastic disease. In this study, we aimed to determine the most specific histopathological and immunohistochemical features required for accurate diagnosis that can reliably predict the clinical behavior. Methods: Sixty-five cases of products of conception were reviewed clinically and pathologically, and any progression to persistent gestational trophoblastic disease (GTD), if present, was noted. Pathological assessment of the archival material included re-cut sections of $5 \mu \mathrm{m}$ in thickness, routine staining with hematoxylin and eosin and immunohistochemical staining of p57Kip2. Results: Certain histopathological criteria were found to be significant in differentiation between complete hydatidiform mole (CHM) and partial hydatidiform mole including villous shape and outline, villous trophoblast hyperplasia, and atypia in extravillous trophoblasts. There were no significant differences in any morphological or immunohistochemical features between cases with or without subsequent development of GTD. Conclusions: Histopathological diagnosis of molar pregnancy remains problematic especially in early gestation. Their diagnosis should be stated after a constellation of specific histopathological criteria in order not to miss CHM. p57Kip2 immunohistochemistry is of great value in diagnosis of cases that had equivocal morphology by histopathological examination. However, there were no significant features to predict cases that subsequently developed persistent GTD.
\end{abstract}

Key Words: Hydatidiform mole; Complete hydatidiform mole, early; p57Kip2 immunohistochemistry
Hydatidiform mole (HM) is an abnormal gestation characterized by significant hydropic change and variable trophoblastic proliferation involving part or all of chorionic villi. ${ }^{1,2} \mathrm{HM}$ is categorized into two separate entities, complete hydatidiform mole (CHM) and partial hydatidiform mole (PHM), according to morphology and cytogenetics. However, hydropic abortion (HA) could morphologically mimic HM.,

Characteristic morphologic features have been proposed for the diagnosis of product of conception (POC) specimens including CHM, PHM, and HA. However, substantial histopathological similarities are observed between the three entities, especially in cases of early detection resulting in interobserver and intraobserver variability in the diagnosis of POC specimens. ${ }^{5,6}$ Thus, the diagnosis of POC on the basis of histopathology alone remains a challenge for pathologists, even those experienced. ${ }^{7}$

Persistent gestational trophoblastic disease (PGTD) develops after CHM in $10 \%$ to $30 \%$ of cases, ${ }^{4}$ and after PHM in $0.5 \%$ to $5 \%$ whereas HA has no relation to PGTD. ${ }^{5}$ Thus, follow-up serum $\beta$-human chorionic gonadotropin ( $\beta$-HCG) measurements aren't essential in cases of $\mathrm{HA}$, whereas these measurements form part of surveillance for PGTD in cases of HM. Therefore, accurate distinction of CHM from PHM on one hand and of HM from HA on the other hand is important for appropriate clinical management and has prognostic implications. ${ }^{3}$

Difficult cases may require molecular techniques using the differences in DNA content of different POC cases. ${ }^{8}$ However, such molecular diagnostic methods are technically difficult, relatively costly, and non-available in most of pathology laboratories. ${ }^{2}$ Detection of the expression of gene products such as $\mathrm{p} 57 \mathrm{Kip} 2$ by the trophoblastic cells should be highlighted. The p57Kip2 gene (CDKN1C) is a strongly paternally imprinted gene expressed only by the maternal allele in most tissues and is involved in implan- 
tation. ${ }^{9}$ In normal placenta, nuclear p57Kip2 expression is seen in villous cytotrophoblast, extravillous trophoblast, villous stromal cells, and deciduas. ${ }^{10,11}$ p57Kip2 can identify CHM (androgenetic diploidy) by the lack of its expression ${ }^{12-14}$ and can be helpful in distinguishing CHM from PHM and non molar HA, ${ }^{11}$ but can't distinguish PHM (diandric monogynic triploidy) from nonmolar (biparental diploidy) specimens as both are positive. ${ }^{14} \mathrm{CHM}$ mostly doesn't contain maternal genome. Therefore, p57Kip2 staining is unexpressed or greatly reduced in the nuclei of their cytotrophoblasts and stromal cells. ${ }^{11}$ Products of conception containing maternal genetic material, PHM and nonmolar HA, show positive nuclear $\mathrm{p} 57 \mathrm{Kip} 2$ staining in cytotrophoblast and villous stromal cells. p57Kip2 is also expressed in intermediate trophoblast islands and decidual cells, serving as positive internal control in all POC cases. ${ }^{12,13}$

In this study, we aimed to define precise histopathological and immunohistochemical features to make a proper diagnosis of the different types of HMs especially in early gestational ages and to distinguish those from other mimics. Furthermore, specific features to predict prognosis and progression to PGTD were investigated.

\section{MATERIALS AND METHODS}

\section{Sample collection}

Archival materials of sixty five cases of POC specimens were retrieved from the department of Pathology, Faculty of Medicine, Mansoura University, between January 2013 and December 2014. The protocol was approved by the Ethical Committee of Mansoura University and informed consents were obtained from the patients.

\section{Histopathological review}

The paraffin blocks were re-cut in 5- $\mu \mathrm{m}$-thick sections and stained with hematoxylin and eosin stain and were independently reviewed by two pathologists for evaluation of the main morphological findings of $\mathrm{HM} .^{13,15}$

\section{Immunohistochemistry}

Five-micrometer-thick tissue section from each case was subject to immunohistochemical staining using monoclonal antibody against the p57Kip2 protein (NeoMarkers/Lab Vision Corporation, Fremont, CA, USA) with dilution 1:200 and the Envision system (DakoCytomation, Glostrup, Denmark). All the steps were performed according to the manufacturers' instructions.

In all cases, p57Kip2 was assessed in the nuclei of the villous cytotrophoblasts, extravillous trophoblasts, and villous stromal cells. Specimens were interpreted as positive for $\mathrm{p} 57 \mathrm{Kip} 2$ staining when there was distinct nuclear staining of villous cytotrophoblasts and stromal cells. The p57Kip2 stain was interpreted as negative when there was no distinct staining or limited nuclear staining $(<10 \%)$ of villous cytotrophoblasts and stromal cells. Staining of intermediate trophoblasts and/or maternal decidua was considered as the positive internal control for these specimens.

\section{Statistical methods}

Data were analyzed using the program SPSS ver. 20 (IBM Corp., Armonk, NY, USA) to obtain descriptive statistics. Statistical significance was determined at $95 \%$ level of confidence (i.e., differences will be considered significant if $\mathrm{p}<.05$ ).

\section{RESULTS}

\section{Examination of clinical data}

The age of patients ranged from 16 to 50 years with a mean of $26 \pm 8$ years and a median of 24 years. All cases were in the first or early second trimester. Their gestational age ranged from 6 to 14 weeks with a mean of $9 \pm 2$ weeks and a median of 8 weeks.

\section{Histopathological examination}

Significant histopathological criteria for the diagnosis and distinguishing different entities are shown in Table 1. Examples of the morphological features are demonstrated in Fig. 1.

\section{Immunohistochemistry}

Specimens that showed distinct nuclear staining of cytotrophoblasts and villous stromal cells were interpreted as positive for p57Kip2 (Fig. 1H). On the other hand, specimens that showed no or limited nuclear staining (in $<10 \%$ ) of cytotrophoblasts

Table 1. Significant histopathological criteria according to the final diagnosis of the studied cases using p57Kip2 immunohistochemistry

\begin{tabular}{ll}
\hline $\begin{array}{l}\text { Significant criteria differentiating } \\
\text { between } \mathrm{CHM} \text { and PHM }\end{array}$ & $\begin{array}{l}\text { Significant criteria differentiating } \\
\text { between molar and nonmolar } \\
\text { pregnancy }\end{array}$ \\
\hline $\begin{array}{l}\text { Villous shape and outline: } p<.001 \\
\text { Villous trophoblast hyperplasia: }\end{array}$ & $\begin{array}{l}\text { Villous shape and outline: } p<.001 \\
p=.001\end{array}$ \\
$\begin{array}{l}\text { Atypia at extravillous trophoblast: } \\
p<.001\end{array}$ & Trophophoblastic inclusion: $p=.001$ \\
& Villous trophoblast hyperplasia: \\
& $p<.001$ \\
\hline
\end{tabular}

$\mathrm{CHM}$, complete hydatidiform mole; PHM, partial hydatidiform mole. 


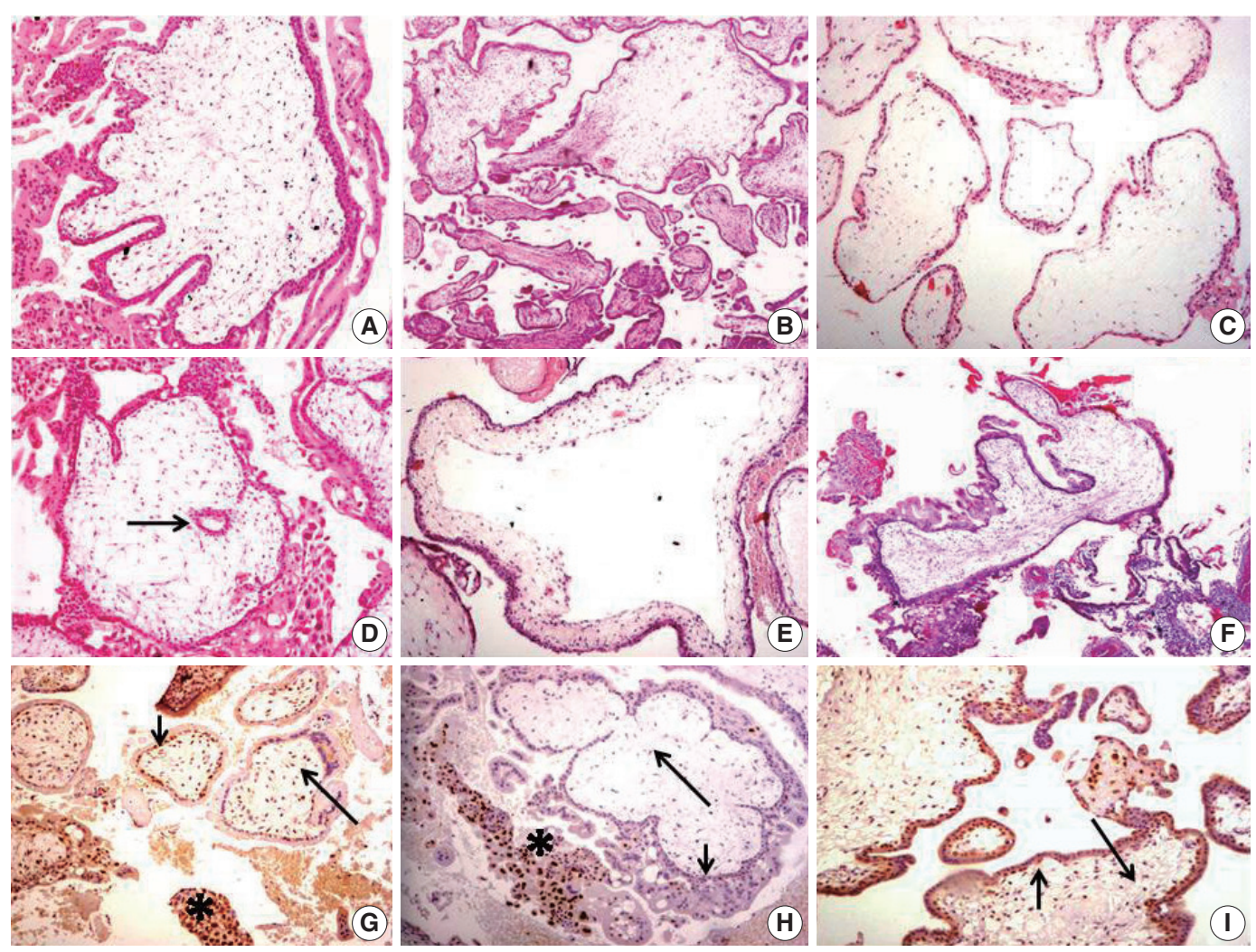

Fig. 1. (A) Complete hydatidiform mole (CHM): irregular villous outline (club shaped) with villous stromal karyorrhectic debris. (B) Partial hydatidiform mole (PHM). Two villous populations; large edematous villi with irregular outline and normal appearing nondistended ones. (C) Hydropic abortion (HA): distended villi with hydropic change. (D) CHM: trophoblastic inclusion (arrow). (E) CHM: cistern formation. (F) CHM: multifocal villous trophoblastic hyperplasia. (G) HA: positive p57Kip2 nuclear staining in intermediate trophoblast as positive internal control (asterisk). Positive p57Kip2 nuclear staining in villous cytotrophoblasts (CT) (short arrow) and villous stromal cells (long arrow) (immunoperoxidase). (H) CHM p57Kip2 immunohistochemistry: negative nuclear staining in villous CT (short arrow) and villous stromal cells (long arrow); positive p57Kip2 nuclear staining in intermediate trophoblast as positive internal control (asterisk) (immunoperoxidase). (I) PHM: positive p57Kip2 nuclear staining in villous CT (short arrow) and villous stromal cells (long arrow) (immunoperoxidase).

and villous stromal cells with positive internal control were considered negative for p57Kip2 stain (Fig. 1). Accordingly, the studied cases were redistributed as shown in Table 2.

$\mathrm{CHM}$ originally constituted 39 cases. However, after reevaluation based on the histopathological criteria and p57Kip2 immunohistochemistry, seven cases were added to CHM (previously diagnosed as PHM). On the other hand, one case originally diagnosed as CHM was converted to PHM. The final typing of the molar cases was, therefore, 45 cases of CHM and 11 cases of PHM.

\section{Follow-up}

By following the clinical history of all studied cases, nine cases (14\% of total/16\% of molar cases) were found to progress into gestational trophoblastic disease (GTD) based on persistence of symptoms and serum $\beta$-HCG level (Table 3). These represented $18 \%$ of CHM and $9 \%$ of PHM cases. As shown in Table 4, histopathological criteria of these cases were examined and compared
Table 2. Comparison of the diagnosis of the studied cases by histopathological examination and p57Kip2 immunohistochemistry ( $\mathrm{n}=$ 56)

\begin{tabular}{lccc}
\hline \multirow{2}{*}{ Method of diagnosis } & \multicolumn{2}{c}{ Case } & \multirow{2}{*}{ p-value } \\
\cline { 2 - 3 } & CHM & PHM & \\
\hline Haematoxylin and eosin stain & $39(60)$ & $17(26)$ & $<.001$ \\
p57Kip2 immunohistochemistry & $45(69)$ & $11(17)$ & \\
\hline
\end{tabular}

Values are presented as number (\%).

$\mathrm{CHM}$, complete hydatidiform mole; PHM, partial hydatidiform mole.

with the results of p57Kip2 immunohistochemistry to detect the most statistically significant histopathological feature that can predict the progression of molar disease into PGTD. According to the current study, none of the studied histopathological parameters could differentiate between cases with or without progression to GTD. 
Table 3. Cases that developed gestational trophoblastic neoplasia $(n=9)$

\begin{tabular}{lcccccccc}
\hline Case & Age $(\mathrm{yr})$ & $\mathrm{GA}(\mathrm{wk})$ & Histopathology & p57Kip2 & $\beta$-HCG $(\mathrm{mlU} / \mathrm{mL})$ & MTX & Response & Second-line treatment \\
\hline 1 & 20 & 10 & CHM & Negative & 2907 & 2 & Yes & - \\
2 & 28 & 4 & CHM & Negative & 1294 & 2 & Yes & - \\
3 & 29 & 8 & CHM & Negative & 280 & 1 & Yes & - \\
4 & 48 & 8 & CHM & Negative & 1328 & 3 & No & Hysterectomy \\
5 & 20 & 12 & CHM & Negative & 4177 & 3 & Yes & - \\
6 & 29 & 9 & CHM & Negative & 446 & 2 & Yes & - \\
7 & 19 & 8 & CHM & Negative & 3260 & 2 & Yes & - \\
8 & 42 & 10 & CHM & Negative & 5000 & 6 & No & EMACO \\
9 & 24 & 8 & PHM & Positive & 4990 & 5 & Yes & - \\
\hline
\end{tabular}

GA, gestational age; $\beta$-HCG, $\beta$-human chorionic gonadotropin; MTX, methotrexate; CHM, complete hydatidiform mole; EMA/CO, etoposide, methotrexate, actinomycin D, cyclophosphamide, vincristine/oncovine; PHM, partial hydatidiform mole.

Table 4. Histopathological criteria of cases that clinically progressed to PGTD in comparison to the rest of the series

\begin{tabular}{|c|c|c|c|c|}
\hline Parameters & & $\operatorname{PGTD}(n=9)$ & Non-PGTD $(n=56)$ & $p$-value \\
\hline \multirow[t]{4}{*}{ Villous shape and outline } & Distended regular & 1 & 9 & $>.99$ \\
\hline & Distended irregular & 5 & 24 & \\
\hline & Non-distended & 1 & 8 & \\
\hline & Two population of villi (distended and nondistended) & 2 & 15 & \\
\hline \multirow[t]{2}{*}{ Cistern } & Present & 8 & 45 & $>.99$ \\
\hline & Absent & 1 & 11 & \\
\hline \multirow[t]{2}{*}{ Trophoblastic inclusion } & Present & 6 & 34 & $>.99$ \\
\hline & Absent & 3 & 22 & \\
\hline \multirow[t]{2}{*}{ Stromal myxoid change } & Present & 4 & 26 & $>.99$ \\
\hline & Absent & 5 & 30 & \\
\hline \multirow[t]{3}{*}{ Villous trophoblast hyperplasia } & Circumfrential & 4 & 21 & $>.99$ \\
\hline & Multifocal & 4 & 27 & \\
\hline & Polar & 1 & 8 & \\
\hline \multirow[t]{2}{*}{ Stromal karyorrhexis } & Present & 0 & 14 & .186 \\
\hline & Absent & 9 & 42 & \\
\hline \multirow[t]{2}{*}{ Atypia at extravillous trophoblast } & Mild & 5 & 32 & $>.99$ \\
\hline & Marked & 4 & 24 & \\
\hline
\end{tabular}

PGTD, persistent gestational trophoblastic disease.

\section{DISCUSSION}

The present study was performed to investigate histopathological parameters commonly used during routine histopathological examination in the differential diagnosis of POC cases, especially those with early gestational age. Also, we aimed to study the value of using these histopathological criteria for diagnosis of HM specimens by comparing them with the results of $\mathrm{p} 57 \mathrm{Kip} 2$ immunohistochemical staining.

In agreement with previous reports, our results showed that examination of the villous shape was statistically significant in differentiation between CHM and PHM cases on one hand and between molar and nonmolar cases on the other hand. ${ }^{16,17}$ Most of our studied CHM cases (62\%) had a population of distended villi with irregular outline. This was significant in differentiation between POC specimens and in favor of early CHM. ${ }^{15,18}$ Our results were in contrast with the results of the study by Ishikawa et al..$^{16}$ who found that enlarged villi with regular round outline was a good marker for the diagnosis of CHM. This disagreement can be explained by the fact that the gestational age of our cases was in the first trimester, and at this early gestational age, villi of CHM don't exhibit the well formed distended villi with regular outline and have more irregular outline.

Cistern formation is a major criterion in HM. Our study showed that cistern formation was found in CHM (96\%) more common than in PHM cases (91\%). It was significant in differentiation between molar and nonmolar pregnancy. On the other hand, it wasn't significant in differentiation between the CHM and PHM. This is in contrast to other studies that found it significant and in favor of diagnosis of PHM. ${ }^{17,18}$ This may be because of the difference in number of studied CHM cases.

We found that the presence of trophoblastic inclusion in the 
villous core was present in $82 \%$ of $\mathrm{PHM}$ and in 67\% of $\mathrm{CHM}$. This was statistically significant in differentiation between cases of molar and nonmolar pregnancy but was insignificant in differentiation between the CHM and PHM as described in previous reports. ${ }^{16,17,19}$ Trophoblastic hyperplasia is an essential requirement for the diagnosis of molar pregnancy. ${ }^{20}$ The results of this study demonstrated that $59 \%$ of $\mathrm{CHM}$ cases had circumferential villous trophoblastic hyperplasia, while $88 \%$ of PHM cases exhibited multifocal villous trophoblast hyperplasia. Polar trophoblast hyperplasia was in favor of nonmolar POC diagnosis. It was found to be statistically significant in differentiation between $\mathrm{CHM}$ and PHM on one hand and between molar and nonmolar POC on the other hand, in agreement with previous studies. ${ }^{19,20} \mathrm{On}$ the other hand, our results disagreed with the study of Abdou et al. ${ }^{17}$ on 59 cases of POC specimens and found that trophoblast hyperplasia had no statistically significant value in that differentiation. This marked circumferential villous trophoblast hyperplasia which was found in CHM cases may be related to the absence of p57Kip2 expression by the villous cytotrophoblasts and villous stromal cells in $\mathrm{CHM}$ cases which lack the maternal genome. This results in loss of cell cycle control and hence increased trophoblast proliferation.

It is known that villi in CHM have a high level of stromal karyorrhectic debris. ${ }^{21}$ In this study, villous stromal karyorrhectic debris wasn't observed in any case diagnosed as PHM or nonmolar POC. Although the villous stromal karyorrhectic debris was found in $36 \%$ of CHM cases, it wasn't statistically significant in differentiation of the three entities; $\mathrm{CHM}$ vs PHM or molar vs nonmolar pregnancies. This was in agreement with the finding that stromal karyorrhexis couldn't differentiate between POC cases. ${ }^{17}$ This was in disagreement with the results of a study including 113 specimens of POC before 13th week gestation and found that the rate of stromal karyorrhexis was significantly higher in early CHM than in PHM $(\mathrm{p}<.001)$. They proved and confirmed that the frequent karyorrhexis in the villous stroma is a useful histopathologic parameter in the differential diagnosis of CHM from PHM and HA in early gestational age. However, the stromal karyorrhexis couldn't be used as a significant feature in cases showing diffuse hydropic change due to an absence of cellular components in the stroma. ${ }^{18}$

The current study showed that atypia (in the form of increased nucleocytoplasmic ratio, hyperchromasia, and pleomorphism) in extravillous trophoblast was mainly of marked degree in $74 \%$ of $\mathrm{CHM}$, and of mild degree in $71 \%$ of PHM cases. Marked atypia was in favor of $\mathrm{CHM}$ diagnosis, while mild atypia was in favor of PHM and HA diagnosis. Marked degree of atypia was statistically significant in differentiation between $\mathrm{CHM}$ and
PHM specimens, while mild degree of atypia wasn't significant in differentiation between molar and nonmolar POC. It may be a useful histopathologic feature regarding the diagnosis and classification of HM. This was consistent with the observation that trophoblast atypia could differentiate between CHM and PHM where diffuse marked atypia was found in most $\mathrm{CHM}$ and focal mild atypia in most PHM. ${ }^{22}$ This was in contrast with others who found that atypia in extravillous trophoblast showed no significant value in differentiation between CHM and PHM. ${ }^{17,19}$

In our study, seven cases diagnosed histopathologically as PHM were reclassified as CHM after p57Kip2 immunohistochemistry (showed no or scattered nuclear staining in villous CT and villous stromal cells). Six of these cases exhibited morphological features similar to PHM on histopathological examination in the form of two populations of villi; small and large distended villi with irregular outline and multifocal villous trophoblast hyperplasia. One case showed two villous populations, circumferential villous trophoblast hyperplasia and trophoblastic inclusion in the core of their villi without stromal karyorrhexis. In these cases, we can consider p57Kip2 as a gold standard for the diagnosis and classification of HM cases. This was similar to previous observations. $^{10}$

In a retrospective study done by Landolsi $e t a l^{2}$ on 220 specimens of HA, negative p57Kip2 expression was observed in 8 cases with a histopathological diagnosis of PHM, and in one case with a diagnosis of HA. Landolsi et al. ${ }^{2}$ tried to explain this negative p57Kip2 expression either due to mis-diagnosis of CHM or lack of staining due to loss of antigenicity. They proved the mis-diagnosis of CHM by genotyping analysis of their discordant nine cases and found absence of their maternal allele. ${ }^{2}$ Funkunaga et al. ${ }^{7}$ reported artifactual loss of staining due to loss of antigenicity in four HA and one PHM cases, but the presence of positive internal control in decidual and implantation site trophoblasts in our study excludes this explanation. ${ }^{12}$

In the current study, one case histopathologically diagnosed as $\mathrm{CHM}$ was inconsistent with the pattern of p57Kip2 immunostaining which showed positive nuclear staining in villous $\mathrm{CT}$ and villous stromal cells. This was also found in the results of previous studies in which one case out of $132 \mathrm{CHM}$ cases was found to show positive p57Kip2 immunostaining in their CT and villous stromal cells. ${ }^{2}$ This was explained by false-positive immunohistochemistry or a mis-diagnosis of PHM or HA. Another possibility is the presence of twin gestation, one of them normal and the other CHM. Furthermore, rare CHMs are biparental in origin and contain both maternal and paternal chromosomal components. They explained it as a mis-diagnosis, as no adequate 
DNA material was available for genetic study to confirm. In such rare cases, we can't consider p57Kip2 as a gold standard for the diagnosis and the matter will require further studies including molecular techniques using the differences in DNA content.

Concerning the follow-up data, nine cases out of the 65 total cases (14\%) developed PGTD. They were diagnosed based on persistence of their symptoms and their serum $\beta$-HCG level which didn't come down to the basal level. Eight cases of these were diagnosed as CHM (18\% of total CHM). Similar results were found in a study performed by Van Cromvoirt et al..$^{23}$ to identify cases the developed PGTD after CHM; 89 cases (20\%) of their total 448 CHM cases developed PGTD and required chemotherapy. ${ }^{24-26}$

According to our results, one case of PHM (9\% of total PHM) had developed PGTD. But this was inconsistent with the percentage detected in a study done by Wielsma et al..$^{24}$ which detected cases of PHM that developed PGTD; only 6 out of 344 PHM cases $(2 \%)$ were found to have developed PGTD and were treated successfully by methotrexate chemotherapy. Also, it was inconsistent with the percentage reported by Chen $e t a l .^{3}$ who found that $2.5 \%-7.5 \%$ of PHM can progress to PGTD.We can attribute this difference between the two studies to the very limited number of the PHM cases studied in the current study. We reviewed these cases histopathologically and didn't find any specific morphological or immunohistochemical features associated with their progression to PGTD. In that regard, the current study was in agreement with the histopathological and immunohistochemical study done by Petts et al..$^{25}$ on 150 cases of molar pregnancy. To achieve this distinction, further studies are required using a larger number of cases and implementing further immunohistochemical markers that may have a benefit .

In conclusion, histopathological diagnosis of molar pregnancy remains problematic especially in early gestational age. The diagnosis requires a constellation of specific histopathological criteria in order not to miss the diagnosis of CHM. p57Kip2 immunohistochemistry is of great value in the diagnosis of cases that have equivocal morphology by histopathological examination. However, there are no significant features to predict cases that subsequently develop persistent trophoblastic disease. Further immunohistochemical markers should be studied to accurately distinguish PHM from CHM and also to predict patients' outcome.

\section{Conflicts of Interest}

No potential conflict of interest relevant to this article was reported.

\section{REFERENCES}

1. Cheung AN. Gestational trophoblastic disease. In: Robboy SJ, Mutter GL, Prat J, Bentley RC, Russell P, Anderson MC, eds. Robboy's pathology of the female reproductive tract. Edinburgh: Churchill Livingstone Elsevier, 2009; 881-907.

2. Landolsi H, Missaoui N, Brahem S, Hmissa S, Gribaa M, Yacoubi MT. The usefulness of p57(KIP2) immunohistochemical staining and genotyping test in the diagnosis of the hydatidiform mole. Pathol Res Pract 2011; 207: 498-504.

3. Chen KH, Hsu SC, Chen HY, Ng KF, Chen TC. Utility of fluorescence in situ hybridization for ploidy and p57 immunostaining in discriminating hydatidiform moles. Biochem Biophys Res Commun 2014; 446: 555-60.

4. Chiang S, Fazlollahi L, Nguyen A, Betensky RA, Roberts DJ, Iafrate AJ. Diagnosis of hydatidiform moles by polymorphic deletion probe fluorescence in situ hybridization. J Mol Diagn 2011; 13: 406-15.

5. Berkowitz RS, Goldstein DP. Clinical practice: molar pregnancy. N Engl J Med 2009; 360: 1639-45.

6. Vang R, Gupta M, Wu LS, et al. Diagnostic reproducibility of hydatidiform moles: ancillary techniques (p57 immunohistochemistry and molecular genotyping) improve morphologic diagnosis. Am J Surg Pathol 2012; 36: 443-53.

7. Fukunaga M, Katabuchi H, Nagasaka T, Mikami Y, Minamiguchi S, Lage JM. Interobserver and intraobserver variability in the diagnosis of hydatidiform mole. Am J Surg Pathol 2005; 29: 942-7.

8. Murphy KM, Ronnett BM. Molecular analysis of hydatidiform moles: utilizing p57 immunohistochemistry and molecular genotyping to refine morphologic diagnosis. Pathol Case Rev 2010; 15: 126-34.

9. Popiolek DA, Yee H, Mittal K, et al. Multiplex short tandem repeat DNA analysis confirms the accuracy of p57(KIP2) immunostaining in the diagnosis of complete hydatidiform mole. Hum Pathol 2006; 37: $1426-34$.

10. Banet N, DeScipio C, Murphy KM, et al. Characteristics of hydatidiform moles: analysis of a prospective series with p57 immunohistochemistry and molecular genotyping. Mod Pathol 2014; 27: 23854.

11. Sarmadi S, Izadi-Mood N, Abbasi A, Sanii S. p57KIP2 immunohistochemical expression: a useful diagnostic tool in discrimination between complete hydatidiform mole and its mimics. Arch Gynecol Obstet 2011; 283: 743-8.

12. Madi JM, Braga AR, Paganella MP, Litvin IE, Da Ros Wendland EM. Accuracy of p57KIP2 compared with genotyping for the diagnosis of complete hydatidiform mole: protocol for a systematic review and meta-analysis. Syst Rev 2016; 5: 169. 
13. Buza N, Hui P. New diagnostic modalities in the histopathological diagnosis of hydatidiform moles. Diagn Histopathol 2012; 18: 201-9.

14. Khooei A, Atabaki Pasdar F, Fazel A, et al. Ki-67 expression in hydatidiform moles and hydropic abortions. Iran Red Crescent Med J 2013; 15: 590-4.

15. Clement PB, Young RH. Atlas of gynaecologic surgical pathology. 3rd ed. London: Elsevier, 2014; 271-97.

16. Ishikawa N, Harada Y, Tokuyasu Y, Nagasaki M, Maruyama R. Reevaluation of the histological criteria for complete hydatidiform mole: comparison with the immunohistochemical diagnosis using p57KIP2 and CD34. Biomed Res 2009; 30: 141-7.

17. Abdou A, Kandil M, El-Wahed MA, Shabaan M, El-Sharkawy M. The diagnostic value of $\mathrm{p} 27$ in comparison to $\mathrm{p} 57$ in differentiation between different gestational trophoblastic diseases. Fetal Pediatr Pathol 2013; 32: 395-411.

18. Kim MJ, Kim KR, Ro JY, Lage JM, Lee HI. Diagnostic and pathogenetic significance of increased stromal apoptosis and incomplete vasculogenesis in complete hydatidiform moles in very early pregnancy periods. Am J Surg Pathol 2006; 30: 362-9.

19. Landolsi H, Missaoui N, Yacoubi MT, et al. Assessment of the role of histopathology and DNA image analysis in the diagnosis of molar and non-molar abortion: a study of 89 cases in the center of Tunisia. Pathol Res Pract 2009; 205: 789-96.

20. Bajaj MS, Mehta M, Kashyap S, et al. Clinical and pathologic profile of angiomyxomas of the orbit. Ophthal Plast Reconstr Surg 2011;
27: 76-80.

21. Chiu PM, Ngan YS, Khoo US, Cheung AN. Apoptotic activity in gestational trophoblastic disease correlates with clinical outcome: assessment by the caspase-related M30 CytoDeath antibody. Histopathology 2001; 38: 243-9.

22. Montes M, Roberts D, Berkowitz RS, Genest DR. Prevalence and significance of implantation site trophoblastic atypia in hydatidiform moles and spontaneous abortions. Am J Clin Pathol 1996; 105: 411-6.

23. van Cromvoirt SM, Thomas CM, Quinn MA, McNally OM, Bekkers RL. Identification of patients with persistent trophoblastic disease after complete hydatidiform mole by using a normal 24-hour urine hCG regression curve. Gynecol Oncol 2014; 133: 542-5.

24. Wielsma S, Kerkmeijer L, Bekkers R, Pyman J, Tan J, Quinn M. Persistent trophoblast disease following partial molar pregnancy. Aust N Z J Obstet Gynaecol 2006; 46: 119-23.

25. Petts G, Fisher RA, Short D, Lindsay I, Seckl MJ, Sebire NJ. Histopathological and immunohistochemical features of early hydatidiform mole in relation to subsequent development of persistent gestational trophoblastic disease. J Reprod Med 2014; 59: 213-20.

26. Hemida R, Arafa M, AbdElfattah H, Sharaf-Eldin D. Human chorionic gonadotropin (hCG) testing in specimens of tumor and myometrial tissues during surgical treatment of gestational trophoblastic tumors. J Cancer Res Updates 2015; 4: 122-6. 